\title{
Optimized Entanglement Purification
}

\author{
Stefan Krastanov ${ }^{1,2}$, Victor V. Albert ${ }^{1,2,3}$, and Liang Jiang ${ }^{1,2}$ \\ ${ }^{1}$ Departments of Applied Physics and Physics, Yale University, New Haven, CT 06511, USA \\ ${ }^{2}$ Yale Quantum Institute, Yale University, New Haven, CT 06520, USA \\ ${ }^{3}$ Walter Burke Institute for Theoretical Physics and Institute for Quantum Information and Matter, California Institute of \\ Technology, Pasadena, California 91125, USA \\ February 15, 2019
}

We investigate novel protocols for entanglement purification of qubit Bell pairs. Employing genetic algorithms for the design of the purification circuit, we obtain shorter circuits achieving higher success rates and better final fidelities than what is currently available in the literature. We provide a software tool for analytical and numerical study of the generated purification circuits, under customizable error models. These new purification protocols pave the way to practical implementations of modular quantum computers and quantum repeaters. Our approach is particularly attentive to the effects of finite resources and imperfect local operations phenomena neglected in the usual asymptotic approach to the problem. The choice of the building blocks permitted in the construction of the circuits is based on a thorough enumeration of the local Clifford operations that act as permutations on the basis of Bell states.

The eventual construction of a scalable quantum computer is bound to revolutionize both how we solve practical problems like quantum simulation, and how we approach foundational questions ranging from topics in computational complexity to quantum gravity. However, numerous engineering hurdles have to be surmounted along the way, as exemplified by today's race to implement practical quantum error-correcting codes. While great many high performing errorcorrecting codes have been constructed by theorists, only recently did experiments start approaching hardware-level error rates that are sufficiently close to the threshold at which codes actually start to help [1, 2]. A promising approach is the modular architecture [3, 4] for quantum computers with implementations based on, among others, superconducting circuits [5], trapped ions [6, 7], or NV centers [8]. The central theme is the creation of a network of small independent quantum registers of few qubits, with connections capable of distributing entangled pairs between nodes $[4,9]$. Such an architecture avoids the difficulty of creating a single complex structure as described in more monolithic approaches and offers a systematic way to minimize undesired crosstalk and residual interactions while scaling the system. Moreover, the same modules might also be used for the design of quantum repeaters for use in quantum communication [10-14].

Experimentally, there have been significant advances in creating entanglement between modules, with demonstrations in trapped ions [6, 15], NV centers [16, 17], neutral atoms [18], and superconducting circuits [5]. However, the infidelity of created Bell pairs is on the order of $10 \%$, while noise due to local gates and measurements can be much lower than $1 \%$. Purification of the entanglement resource will be necessary before successfully employing it for fault-tolerant computation or communication. Although various purification protocols have been proposed [7, 9, 10, 14, 1922 ], there is still a lack of systematic comparison and optimization of purification circuits, as the number of possible designs increases exponentially with the size of the circuits. In this paper we develop tools to generate and compare purification circuits and we present multiple purification protocols outperforming the contenders we test against [7, 9, 14] over a wide range of realistic hardware parameters. We review the notion of an entanglement purification circuit and present our approach to generating and evaluating such circuits. We compare our results to recent pro- 


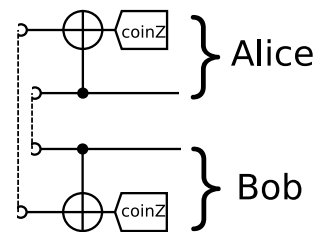

Figure 1: A simple purification circuit of width 2 (i.e. 2 local qubits for Alice or Bob.). The upper half is ran by Alice, while the bottom half is ran by Bob. The dashed lines correspond to the initialization of registers with low-quality "raw" Bell pairs. The top and bottom register correspond to the two qubits of the sacrificial Bell pair. A coincidence measurement in the $Z$ basis marks a successful purification procedure.

posals for practical high-performance purification circuits, and finally discuss the design principles and key ingredients for efficient purification circuits.

Importantly, we pay particular attention to the limitations imposed by working with finite hardware resources. One can find many highly efficient purification schemes in the literature, which reach perfect fidelities at high yield in the asymptotic regime (e.g. [10, 20]), however such asymptotic resource theories neglect the imperfections and size limitations of the purification hardware. Moreover, a large family of such circuits can be constructed from error correcting codes[20, 23], however they can often be impractically wide as they do not exploit the possibility of renewed generation of entanglement in already measured qubit registers. Our work optimizes entanglement purification in today's NISQ [24] devices, complementing the protocols that are optimal only in the asymptotic regime of arbitrarily many available qubits and perfect gates and measurements. The imperfections in the local gates and measurements are the limiting factor in realworld hardware. We compare our results to other known finite protocols (including Oxfords's and IBM's [19, 25], STRINGENT [9, 22], and some recursive or iterative [10] versions of the same).

Purification of Bell Pairs In an entanglement purification protocol, two parties, Alice and Bob, start by sharing a number of imperfect Bell pairs and by performing local gates and measurements and communicating classically, they obtain a single pair of higher fidelity. For conciseness we use A, B, C, and D to denote the Bell basis states.

$$
\begin{aligned}
& A=\left|\phi_{+}\right\rangle=\frac{|00\rangle+|11\rangle}{\sqrt{2}} \\
& B=\left|\psi_{-}\right\rangle=\frac{|01\rangle-|10\rangle}{\sqrt{2}} \\
& C=\left|\psi_{+}\right\rangle=\frac{|01\rangle+|10\rangle}{\sqrt{2}} \\
& D=\left|\phi_{-}\right\rangle=\frac{|00\rangle-|11\rangle}{\sqrt{2}}
\end{aligned}
$$

The imperfect pairs are described in the Bell basis (eq. 1) by $\rho_{0}=F_{0}|A\rangle\langle A|+\frac{1-F_{0}}{3}(|B\rangle\langle B|+| C\rangle\langle C|+$ $|D\rangle\langle D|$ ). If we have a state of multiple pairs (like AA), the first letter will denote the pair to be purified.

To explain the roles of the local gates and coincidence measurements let us consider, in Fig. 1, the simplest purification circuit, in which Alice and Bob share two Bell pairs and sacrifice one of them. One way to explain the circuit is to describe it as an error-detecting circuit: If we start with two perfectly initialized Bell pairs in the state $\mathrm{A}$, then the coincidence measurement will always succeed; however, if an X error (a bit flip) happens on one of the qubits, that error will be propagated by the CNOT operations and it will cause the coincidence measurement to fail (the two qubits will point in opposite directions on the $\mathrm{Z}$ axis). It is important to note that only $\mathrm{X}$ and $\mathrm{Y}$ errors can be detected by this circuit, but not $\mathrm{Z}$ errors (phase flips) as the coincidence measurement can not distinguish A from D states. One needs a circuit running on more than two Bell pairs to address $\mathrm{X}, \mathrm{Y}$, and $\mathrm{Z}$ errors.

For the purpose of designing an optimal purification circuit, it is enlightening to also interpret the local operations in terms of permutations of the basis vectors[26, 27]. The initial state of the 4 qubit system is described by the density matrix $\rho_{0} \otimes \rho_{0}$, or equivalently by the 16 scalars in its diagonal in the Bell basis $\{A A, A B, \ldots, D D\}$. The "mirrored" CNOT operations that both Alice and Bob perform result in a new diagonal density matrix with diagonal entries being a permutation of those of the original density matrix. A coincidence measurement on the $\mathrm{Z}$ axis follows, which results in projecting out half of the possible states, i.e. deleting 8 of the scalars and renormalizing and adding by pairs the other 8 . The permutation operation and coincidence measurement have to be chosen together such that this projection (when the coincidence measurement is successful) results in filtering out many of the lower-probability B, C, and D states. A 
detailed run through this example is given in the supplementary materials.

If we restrict ourselves to finding the best "single sacrifice" circuits, i.e. circuits that sacrifice one Bell pair in an attempt to purify another one, we need to find the best set of permutations and measurements. There are 3 coincidence measurements of interest - coincidence in the $\mathrm{Z}$ basis which selects for $\mathrm{A}$ and $\mathrm{D}$; coincidence in $\mathrm{X}$ basis which selects for $\mathrm{A}$ and $\mathrm{C}$; and anti-coincidence in the $\mathrm{Y}$ basis which selects for A and B. All of those measurements can be implemented as a $\mathrm{Z}$ measurement preceded by a local Clifford operation.

The group of possible permutations is rather complicated. Firstly, all permutations of the Bell basis are Clifford operations because the permutation operation can be written as a permutation on the stabilizers of each state (moreover, we do not have access to all 16! permutations, as only operations local for Alice and Bob are permitted). This restriction permits us to efficiently enumerate all possible permutations and study their performance. The software for performing this enumeration is provided with this manuscript. The enumeration goes as follows [28, 29]. There are 11520 operations in the Clifford group of two qubits $C_{2}$. After exhaustively listing all operations in $C_{2}^{\otimes 2}$ we are left with 184320 unique Clifford operations that act as permutations of the Bell basis of two Bell pairs. Accounting for 16 different operations that change only the global phase of the state (e.g. XX which maps B to -B) we are left with 11520 unique permutations. Restricting ourselves to permutations that map A to A cuts that number by a factor of 4 for each pair, which leaves us with 720 unique restricted permutations. Out of those, 72 operations do not change the fidelity $(72=2 \times 6 \times 6$ correspond to two operations (the identity and SWAP) under the six possible BCD permutations for each pair). The remaining 648 permitted operations perform equally well when purifying against depolarization noise, if they are used with the appropriate coincidence measurement. Half of them can be generated from the mirrored CNOT operation from Fig. 1 together with BCD permutations performed before or after on each of the two pairs. The other half can be generated if we also employ a SWAP gate (such gate can be of importance for hardware implementations that have "hot" communication qubits and "cold" storage qubits[7]). When we break the symmetry of the depolarization noise and use biased noise instead, all of these operations still permit purification, but a small fraction of them significantly outperform the rest. So far, we have only counted purification circuits with 2 local qubits. We may increase the width (i.e., number of local qubits) to boost the performance of the entanglement purification. However, the number of possible purification circuits grows exponentially with not only the length, but also the width of the circuit. Even for relatively small circuits (e.g., length 10 and width 3 ), there will be $>10^{40}$ different configurations, if we use the operations discussed above, which are impossible to exhaustively compare. Therefore, we need an efficient procedure to choose the appropriate permutation operation at each round of our purification protocol.

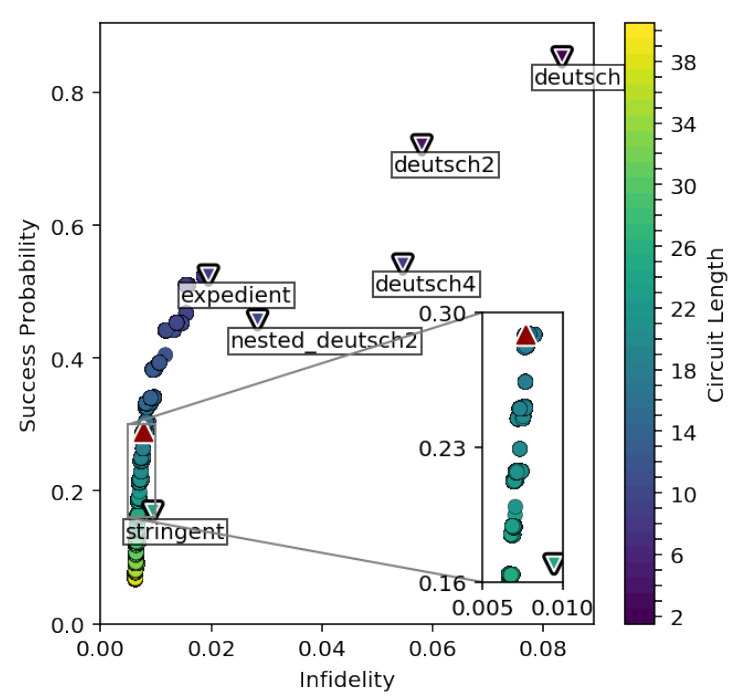

Figure 2: Comparing circuits designed by our genetic algorithm (for three-qubit registers) to prior art. Each circle marks a unique circuit. The horizontal axis is the infidelity of the final pair. The vertical axis is the probability of success. Also shown are the "Oxford" scheme on two Bell pairs [19], which outperforms the IBM scheme [20]. The Innsbruck's "Pumping(2)" and "Pumping(4)" are that same scheme applied consecutively two or four times from [10](Sec 3.a). The aforementioned schemes require two-qubit registers while the rest are for threequbit registers. "Recurrent(2)" is the recursive version (at depth 2) of "Oxford" from[10, 20]. In "Rec.\&Pump." one recursively repeats the pumping protocol instead of the Oxford protocol. To our knowledge "EXPEDIENT" and "STRINGENT" are some of the best circuits $[9,22])$. Evaluations done at $p_{2}=\eta=0.99$ and $F_{0}=0.9$. The red triangle marks a circuit of ours we discuss more in Fig. 3. 
Discrete optimization algorithm The design of circuits, whether quantum or classical, lends itself naturally to the use of evolutionary (or genetic) algorithms with numerous interesting examples in, for instance, electronics and robotics [30]. In particular, in the field of quantum information, such optimization techniques have been used in widely different settings, including control [31], state preparation and metrology [32], and studies of locality as related to Bell's inequality [33]. An evolutionary algorithm is an optimization algorithm particularly useful for cost functions over discrete parameter spaces. A candidate solution (a point in parameter space) plays the role of an individual in a population subjected to simulated evolution. Depending on the particular implementation, each individual generates a number of children, whether through mutations or through sexual modes of reproduction with other individuals in the population. The population is then culled so only the fittest candidate-solutions remain and the procedure is repeated for multiple generations until the convergence criteria are fulfilled.

In our particular implementation ${ }^{1}$ the individuals are quantum entanglement purification circuits. We restrict ourselves to circuits that purify the entanglement between two parties, Alice and Bob, without the involvement of a third party. The circuit can contain any of the previously discussed coincidence measurements (coincidence in $\mathrm{Z}$, coincidence in $\mathrm{X}$, anti-coincidence in $\mathrm{Y}$ ). The circuit is also permitted to contain the "mirrored" CNOT operation from Fig. 1 together with any permutation of the $\{\mathrm{B}, \mathrm{C}, \mathrm{D}\}$ states applied before the CNOT operation. Applying the $\{\mathrm{B}, \mathrm{C}, \mathrm{D}\}$ permutation after the CNOT is unnecessary as the next operation would already have that degree of freedom. However, the final result will have a biased error, so a single $\{\mathrm{B}, \mathrm{C}, \mathrm{D}\}$ permutation at the very end might be required.

\footnotetext{
${ }^{1}$ https://qevo.krastanov.org/ (online repository) The provided software tools and examples in the supplementary materials are readily usable in pedagogical settings as well. Moreover, our software provides analytical expressions for the final fidelities and numerical estimates for the expected resource overhead. The circuits can be fine-tuned during the optimization run for the error model of the particular hardware. This online resource is cloned at krastanov.github.io/qevo/index.html.
}

Operation and measurement errors The design of the purification protocol is sensitive to imperfections in the local operations as well. We parameterize the operational infidelities with the parameters $p_{2}$, where $1-p_{2}$ is the chance for a two-qubit gate to cause a depolarization, and $\eta$, where $1-\eta$ is the chance for a measurement to report the incorrect result.

No memory errors or single-qubit gate errors are considered in our treatment as they are generally much smaller [34, 35], but they can be accounted for in the same manner.

After a measurement the measured qubit pair is reset to a new Bell pair and Alice and Bob can again use it as a purification resource. The initial fidelity of each Bell pair is a parameter $F_{0}$ set at the beginning of the algorithm. Similarly, we set the measurement fidelity $\eta$ and the two-qubit gate fidelity $p_{2}$ at the beginning. More complicated settings with different error models are possible as well - of special interest would be circuits adapted for registers containing a "hot" communication side (e.g. only one qubit in the register is able to establish initial remote entanglement) and a "cold" memory side (considered in Nigmatullin et al. [7]). For that type of registers one would also add the SWAP gate to the permitted genome. However, we show such circuits only in the supplementary materials as the majority of circuits in the literature are designed for registers where all qubits have similar properties.

The "fitness" that we optimize is the fidelity of the final purified Bell pair, however different weights can be placed on the "infidelity components" along $|B\rangle,|C\rangle$, and $|D\rangle$ if needed. In practice, the genetic algorithm is fairly robust to changing parameters like the population size, mutation rates, or number of children circuits. We provide both pregenerated circuits and ready-torun scripts to generate circuits from scratch.

Importantly, depending on the parameter regime and error model, different circuits would be the top performers. This showcases the importance of rerunning the optimization algorithm for the given hardware. An example of such difference is provided in the supplementary materials.

A word of caution: purification yield and finite imperfect circuits A common way to evaluate the performance of a purification circuit is to present its yield, defined as follows. For 
a circuit that starts with $n$ imperfect Bell pairs and that produces $m$ Bell pairs with fidelity arbitrarily close to 1 , the yield is $\lim _{n \rightarrow \infty} \frac{m}{n}$. The limiting procedure is necessary due to the requirement that the final fidelity needs to be arbitrarily close to 1, which is impossible for a finite circuit. In some cases this can be achieved by the recursive (nested) application of a known finite circuit or by the use of the more advanced hashing method [25], as long as the local operations and measurements employed in the circuit are perfect.

However, our focus is on finite circuits of practical interest for near-term hardware. The yield is not a good measure of efficiency in this case as it is defined in terms of a limiting procedure for asymptotic circuits. Moreover, we specifically optimize our circuits to work in the presence of measurement and operational errors, which makes unit fidelities (required for the definition of yield) impossible for a finite circuit. Optimizing in the presence of local errors also makes our circuits better than circuits that would have been optimized for a figure of merit that neglects such errors (the supplementary materials illustrate this with examples of circuits designed for different levels of errors).

We use the final fidelity, the probability of success or the average amount of consumed raw Bell pairs as measurements of efficiency, as these are the quantities of interest in the implementation of small error correcting codes on modular architectures [9] (these quantities decide the delay necessary for the performance of a stabilizer measurement or gate teleportation).

There is an interesting definition of yield that can be used for finite circuits like ours. If one concatenates a finite circuit with the hashing method for purification one can use the yield of the new asymptotic circuit as a figure of merit for the initial finite circuit. This "hashing yield" is still only defined in the presence of perfect measurements and local operations, hence we do not employ it in the main text. However, we discuss it in the supplementary materials.

Lastly, one can relax the requirement in the definition of yield that the final fidelity has to be 1. In that case two new definitions of yield can be considered: $1 / N$ where $N$ is the number of raw Bell pairs that the circuit would require in a best case run ( $N$ is represented as color in Fig. 2) or $1 / N_{\text {avg }}$ where $N_{\text {avg }}$ is how many raw Bell pairs are expended on average until completion (taking into account that the circuit might need to be restarted after a failed measurement). $N_{a v g}$ is calculated through a Monte Carlo simulation and also shown in Fig. 3 for some of the circuits of interest. While $N$ depends only on the particular circuit, $N_{\text {avg }}$ is a function of the error model (e.g. $F_{0}, p_{2}$, and $\eta$ ).

Comparing with Prior Art We generated a few thousand well performing circuits of lengths up to 40 operations and acting on up to six pairs of qubits (and the algorithm can easily generate bigger circuits, but soon one hits a wall in performance due to the imperfections in the local operations as discussed below). The zoo of circuits we have created can be explored online (see note 1), but importantly, one can generate circuits specifically for their hardware using our method. Fig. 2 shows how our circuits compare to a number of other circuits of width 3 . We outperform all circuits in the comparison in terms of final fidelity of the distilled pair, while also having higher probabilities of success, and employing fewer resources or shorter circuits.

In Fig. 3 we compare one of the best performing circuits we know (STRINGENT from [9]) to one particular circuit we have designed. We show only Bob's side of the circuit. Alice performs the same operations and the two parties communicate to perform coincidence measurements. The shading permits us to see which qubit pairs are engaged with other pairs: Each qubit Bob possesses starts with a distinct color; The color is "contagious", i.e. two-qubit gates will "infect" the second qubit in the gate with the color of the first qubit; Measurements followed by regeneration of the raw Bell pair resource reset the color of the measured qubit. The shading clearly shows that the best protocols we find have all the qubits engaged (entangled) together, a finding consistent with the use of "multiple selection" purification protocols introduced in [22]. In contrast, conventional purification protocols have sub-circuits where only a subset of the qubits are engaged together.

A potential caveat of that "completely engaged" approach needs to be addressed: in Fig. 2 we report the probability of all measurement in a given protocol succeeding in a given run, but we do not report the following overhead. If a sin- 
(c)
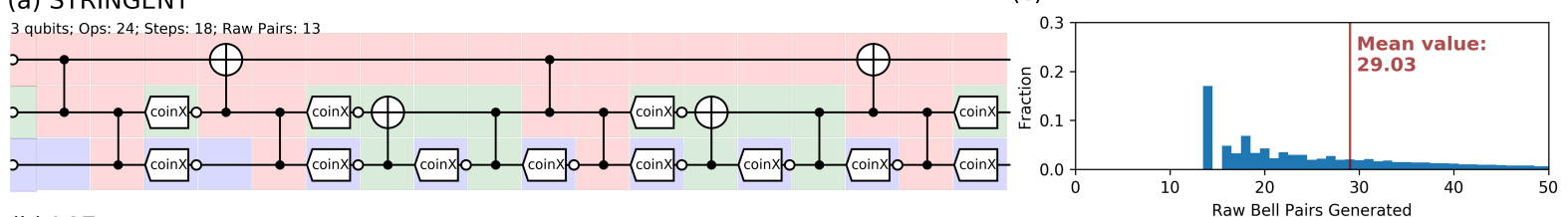

(b) $\mathrm{L} 17$
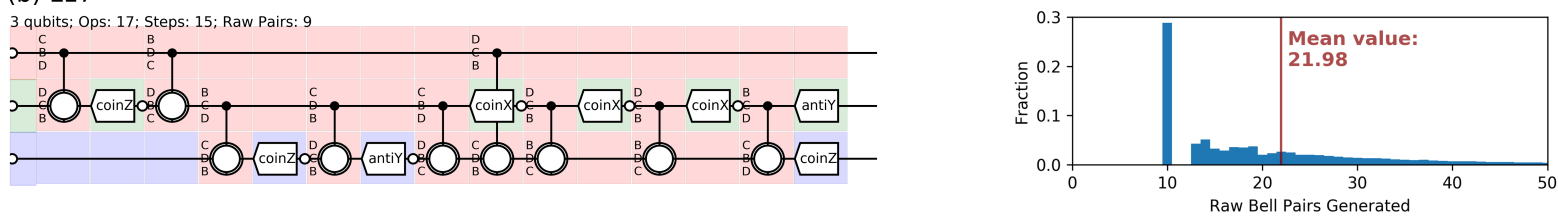

Figure 3: Comparison of (a) the STRINGENT circuit [9] to (b) the L17 circuit obtained through optimization. The L17 circuit outperforms STRINGENT in terms of both final obtained fidelity and success probability over a wide range of error parameters. The color coding shows independent sub-circuits in the STRINGENT circuit and no such sub-circuits in our design. We show only Bob's side of the circuit. The vertical set of letters before each gate marks how the $\{B, C, D\}$ states are permuted, which can be achieved with single qubit gates folded in the CNOT gate as described in the supplementary materials. While we use only CNOT two-qubit gates, we intentionally used a modified symbol in order to bring attention to the presence of these permutation operations. The small white circles after each measurement represent generation of a new Bell pair resource with fidelity $F_{0}$. The histograms (c) are of the required number of Bell pairs for a completion of the protocol (as opposed to a single-shot run) for STRINGENT and the optimized circuit. We also provide analytical evaluations of all our circuits at our online repository. In particular even though STRINGENT is longer (24 operations single shot, 29 raw Bell pairs on average) than L17 (17 operations single shot, 22 raw Bell pairs on average), STRINGENT has higher final infidelity by a factor of $\frac{16}{6} \approx 1.33$ if there are no measurement and operational errors. If we include them, they become the dominant contribution to the final infidelity and in the case of STRINGENT vs L17 the infidelity is still higher by a factor of 1.26 (infidelities of $0.95 \%$ and $0.77 \%$, success probabilities of $17 \%$ and $29 \%$, respectively). The optimization and evaluation was done for $F_{0}=0.9, p_{2}=\eta=0.99$, without memory errors or errors from single-qubit unitaries.

gle measurement fails, the protocol needs to be restarted; the aforementioned conventional purification protocols, that posses sub-circuits, can redo just the failed sub-circuit (for instance, either of the two green blocks in STRINGENT from Fig. 3), instead of restarting the entire circuit. A priori, this might lead to lower resource overhead compared to our protocols (as they generally completely entangle the qubits of each register), even if we still win in terms of final fidelity and probability of success. However, a detailed evaluation of this overhead shows that even when taken into account, our protocols outperform the approaches we compare with as they require lower number of gates, both in best case scenarios and on average (see right panel of Fig. 3).

Our approach can be employed for circuits with more than two sacrificial pairs. Fig. 4 compares the performance of circuits working on 3 pairs (as above) and circuits working on 4 or more pairs of qubits. With still bigger circuits one quickly reaches a fidelity limit imposed by the finite imperfections in the last operations performed by the circuit (for hardware with perfect local opera- tions that limitation does not exist and one would rather use larger circuits that perform many more operations per sacrifice $[21,25,36])$. Example circuits are given online (see note 1 ). For much wider circuits one can surpass this limitation and even use error correcting codes in order to perform perfect (logical) local operations [37, 38], but currently our software is not applicable to these cases.

Operation versus initialization errors The design of efficient purification circuits needs to balance between the initialization errors (imperfect raw Bell pairs) and operation errors (imperfect local gates and measurements). As detailed in the supplementary materials, for arbitrary long purification circuits, the asymptotic infidelity is $\frac{\varepsilon}{2}+\mathcal{O}\left(\varepsilon^{2}\right)$ where $\varepsilon=1-p_{2}$ (as indicated by the vertical dashed line in Fig. 4), which is only limited by the operation errors. For finitelength purification circuits, however, the initialization errors also play an important role, which determines how fast the purification circuits approach the asymptotic limit with increasing cir- 
cuit length (Fig. 4). By analyzing the circuits given by the discrete optimization algorithm, we have observed that: (1) For fixed length, depending on the parameter regime and error model, different circuits would be the top performers. This showcases the importance of rerunning the optimization algorithm for the given hardware; (2) To boost the achievable fidelity, it is important to use double-selection (where two Bell pairs are simultaneously sacrificed to detect errors on a third surviving Bell pair) [22] instead of repeated single-selection (where only one Bell pair is sacrificed at each error detection step). This stems from the fact that the asymptotic infidelity of single-selection is $\frac{7 \varepsilon}{8}$, i.e. nearly twice that of double-selection. Moreover, multiple selection (where $n>2$ Bell pairs are simultaneously sacrificed) has the same dominant asymptotic infidelity of $\frac{\varepsilon}{2}$ as double selection. Therefore, wider circuits provide diminishing returns in the presence of operational errors.

While the operational errors limit the utility of very wide circuits, in the case of perfect local operations this phenomenon of "diminishing returns" does not exist. In that case one can still use our optimization algorithm to design good small circuits, but for large circuits the cost function as currently defined can not be computed efficiently. Another possible direction of interest, which would require the simulation of large circuits, would be the application of discrete optimization algorithms to the purification of multiparty entanglement [9, 39-41], however this might be computationally prohibitive. Of note is, however, that the computational complexity stems from the tracking of the purely classical probabilities at each measurement branch (the circuits are composed of Clifford gates that can be simulated efficiently). We are hopeful that stochastic evaluation of the cost function (through a Monte Carlo method) will be sufficient to surmount any computational challenges in the application of our protocol to larger circuits, however we do not pursue this in the current work.

In conclusion, we have optimized purification circuits of fixed width using a discrete optimization approach using "building-block" subcircuits proven to be optimal. The optimized circuits outperform many other general-purpose purification protocols in all three aspects - fidelity of purified Bell pair, success probability, and circuit length

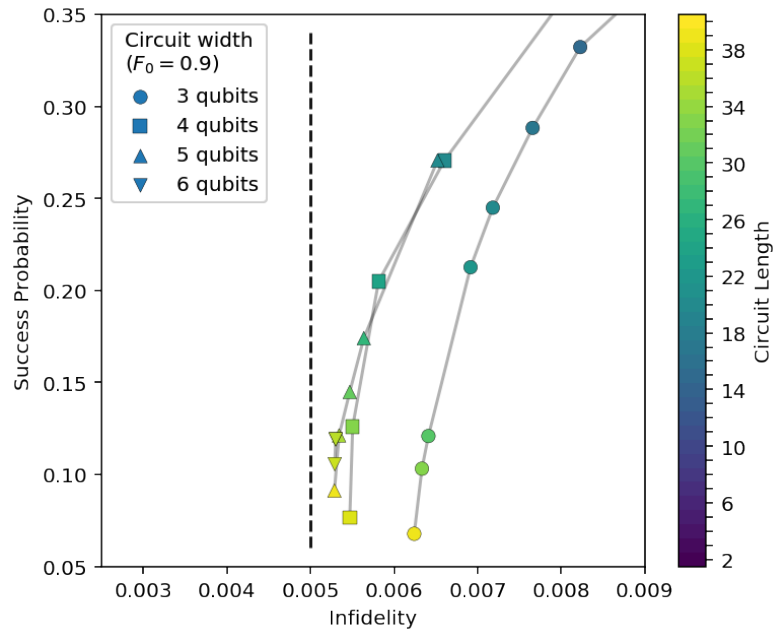

Figure 4: Similarly to Fig. 2 we compare the performance of circuits acting on 3 or more pairs. For legibility, only some of the best generated circuits of each width are shown (evaluated at $F_{0}=0.9$ and $p_{2}=\eta=0.99$ ). Our circuits approach the limit of $\frac{\varepsilon}{2}=0.005$, derived in the main text and supplementary materials.

(whether measured in terms of average number of operations performed or average number of raw Bell pairs used). For purification circuits of width 2 , we analyze the group structure of the Clifford operations that fulfill the locality constraints of purification. For purification circuits of width $\geq 3$, we demonstrate the importance of multiple selection (using at least two sacrificial Bell pairs to simultaneously detect errors), and specify the diminishing returns of using much wider circuits. We numerically obtain efficient purification circuits that approach the asymptotic theoretical limits. Our approach of using discrete optimization algorithms is applicable to various errors models (e.g., dephasing dominated gate errors, imperfect Bell state beyond the Werner form, etc). Moreover, it can be used to optimize the purification circuits in the presence of memory errors, including additional decoherence to all local qubits during the creation of Bell pairs, and to investigate the entanglement purification of encoded Bell pairs.

\section{Acknowledgments}

We are grateful for the helpful input from Holly Mandel and Kyungjoo Noh. This work would not have been possible without the contributions of the Python, Jupyter, Matplotlib, Numpy, Sympy, Qutip, and Julia open source projects and 
the Yale HPC team. We acknowledge support from ARL-CDQI, ARO (W911NF-14-1-0011, W911NF-14-1-0563), ARO MURI (W911NF-161-0349 ), AFOSR MURI (FA9550-14-1-0052, FA9550-15-1-0015), the Alfred P. Sloan Foundation (BR2013-049), and the Packard Foundation (2013-39273).

\section{References}

[1] Julia Cramer, Norbert Kalb, M Adriaan Rol, Bas Hensen, Machiel S Blok, Matthew Markham, Daniel J Twitchen, Ronald Hanson, and Tim H Taminiau. Repeated quantum error correction on a continuously encoded qubit by real-time feedback. Nature communications, 7, 2016. DOI: 10.1038/ncomms11526.

[2] Nissim Ofek, Andrei Petrenko, Reinier Heeres, Philip Reinhold, Zaki Leghtas, Brian Vlastakis, Yehan Liu, Luigi Frunzio, SM Girvin, L Jiang, et al. Extending the lifetime of a quantum bit with error correction in superconducting circuits. Nature, 536(7617):441-445, 2016. DOI: 10.1038/nature18949.

[3] Daniel Gottesman and Isaac L Chuang. Demonstrating the viability of universal quantum computation using teleportation and single-qubit operations. Nature, 402 (6760):390-393, 1999. DOI: 10.1038/46503.

[4] C Monroe, R Raussendorf, A Ruthven, KR Brown, P Maunz, L-M Duan, and J Kim. Large-scale modular quantum-computer architecture with atomic memory and photonic interconnects. Physical Review A, 89(2):022317, 2014. DOI: 10.1103/physreva.89.022317.

[5] A Narla, S Shankar, M Hatridge, Z Leghtas, KM Sliwa, E Zalys-Geller, SO Mundhada, W Pfaff, L Frunzio, RJ Schoelkopf, et al. Robust concurrent remote entanglement between two superconducting qubits. Physical Review X, 6(3):031036, 2016. DOI: 10.1103/physrevx.6.031036.

[6] D Hucul, IV Inlek, G Vittorini, C Crocker, S Debnath, SM Clark, and Cl Monroe. Modular entanglement of atomic qubits using photons and phonons. Nature Physics, 11 (1):37-42, 2015. DOI: 10.1038/nphys3150.

[7] Ramil Nigmatullin, Christopher J Ballance,
Niel de Beaudrap, and Simon C Benjamin. Minimally complex ion traps as modules for quantum communication and computing. New Journal of Physics, 18 (10):103028, 2016. DOI: 10.1088/13672630/18/10/103028.

[8] Andreas Reiserer, Norbert Kalb, Machiel S Blok, Koen JM van Bemmelen, Tim H Taminiau, Ronald Hanson, Daniel J Twitchen, and Matthew Markham. Robust quantum-network memory using decoherence-protected subspaces of nuclear spins. Physical Review X, 6(2):021040, 2016. DOI: 10.1103/physrevx.6.021040.

[9] Naomi H Nickerson, Ying Li, and Simon C Benjamin. Topological quantum computing with a very noisy network and local error rates approaching one percent. $\mathrm{Na}$ ture communications, 4:1756, 2013. DOI: $10.1038 /$ ncomms2773.

[10] W Dür, H-J Briegel, JI Cirac, and P Zoller. Quantum repeaters based on entanglement purification. Physical Review A, 59(1):169, 1999. DOI: 10.1103/physreva.59.169.

[11] Jian-Wei Pan, Christoph Simon, Časlav Brukner, and Anton Zeilinger. Entanglement purification for quantum communication. Nature, 410(6832):1067-1070, 2001. DOI: $10.1038 / 35074041$.

[12] L Childress, JM Taylor, Anders Søndberg Sørensen, and Mikhail D Lukin. Fault-tolerant quantum repeaters with minimal physical resources and implementations based on single-photon emitters. Physical Review A, 72(5):052330, 2005. DOI: 10.1103 /physreva.72.052330.

[13] L Jiang, JM Taylor, and MD Lukin. Fast and robust approach to long-distance quantum communication with atomic ensembles. Physical Review A, 76(1):012301, 2007. DOI: 10.1103/physreva.76.012301.

[14] Naomi H Nickerson, Joseph F Fitzsimons, and Simon C Benjamin. Freely scalable quantum technologies using cells of 5-to-50 qubits with very lossy and noisy photonic links. Physical Review X, 4(4):041041, 2014. DOI: $10.1103 /$ physrevx.4.041041.

[15] DL Moehring, P Maunz, S Olmschenk, KC Younge, DN Matsukevich, L-M Duan, and $\mathrm{C}$ Monroe. Entanglement of singleatom quantum bits at a distance. Nature, 
449(7158):68-71, 2007. DOI: 10.1038/nature06118.

[16] Wolfgang Pfaff, Tim H Taminiau, Lucio Robledo, Hannes Bernien, Matthew Markham, Daniel J Twitchen, and Ronald Hanson. Demonstration of entanglementby-measurement of solid-state qubits. $\mathrm{Na}$ ture Physics, 9(1):29-33, 2013. DOI: 10.1038 /nphys2444.

[17] Bas Hensen, Hannes Bernien, Anaïs E Dréau, Andreas Reiserer, Norbert Kalb, Machiel S Blok, Just Ruitenberg, Raymond FL Vermeulen, Raymond N Schouten, Carlos Abellán, et al. Loophole-free bell inequality violation using electron spins separated by 1.3 kilometres. Nature, 526(7575): 682-686, 2015. DOI: 10.1038/nature15759.

[18] Stephan Ritter, Christian Nölleke, Carolin Hahn, Andreas Reiserer, Andreas Neuzner, Manuel Uphoff, Martin Mücke, Eden Figueroa, Joerg Bochmann, and Gerhard Rempe. An elementary quantum network of single atoms in optical cavities. Nature, 484(7393):195-200, 2012. DOI: 10.1364/icqi.2011.qwb2.

[19] David Deutsch, Artur Ekert, Richard Jozsa, Chiara Macchiavello, Sandu Popescu, and Anna Sanpera. Quantum privacy amplification and the security of quantum cryptography over noisy channels. Physical review letters, 77(13):2818, 1996. DOI: 10.1103/physrevlett.77.2818.

[20] Charles H Bennett, Gilles Brassard, Sandu Popescu, Benjamin Schumacher, John A Smolin, and William K Wootters. Purification of noisy entanglement and faithful teleportation via noisy channels. Physical review letters, 76(5):722, 1996. DOI: 10.1103/physrevlett.76.722.

[21] Wolfgang Dür and Hans J Briegel. Entanglement purification and quantum error correction. Reports on Progress in Physics, 70(8):1381, 2007. DOI: 10.1088/00344885/70/8/r03.

[22] Keisuke Fujii and Katsuji Yamamoto. Entanglement purification with double selection. Physical Review A, 80(4):042308, 2009. DOI: $10.1103 /$ physreva.80.042308.

[23] Hans Aschauer. Quantum communication in noisy environments. PhD thesis, lmu, 2005.

[24] John Preskill. Quantum computing in the nisq era and beyond. arXiv preprint arXiv:1801.00862, 2018. DOI: 10.22331/q2018-08-06-79.

[25] Charles H Bennett, David P DiVincenzo, John A Smolin, and William K Wootters. Mixed-state entanglement and quantum error correction. Physical Review A, 54(5): 3824, 1996. DOI: 10.1103/physreva.54.3824.

[26] Jeroen Dehaene, Maarten Van den Nest, Bart De Moor, and Frank Verstraete. Local permutations of products of bell states and entanglement distillation. Physical Review A, 67(2):022310, 2003. DOI: 10.1103/physreva.67.022310.

[27] H Bombin and MA Martin-Delgado. Entanglement distillation protocols and number theory. Physical Review A, 72(3):032313, 2005. DOI: $10.1103 /$ physreva.72.032313.

[28] Maris Ozols. Clifford group. 2008. DOI: $10.1117 / 12.266854$.

[29] A Robert Calderbank, Eric M Rains, PM Shor, and Neil JA Sloane. Quantum error correction via codes over gf (4). IEEE Transactions on Information Theory, 44(4):1369-1387, $1998 . \quad$ DOI: 10.1109/isit.1997.613213.

[30] Thomas Geijtenbeek, Michiel van de Panne, and A Frank van der Stappen. Flexible muscle-based locomotion for bipedal creatures. ACM Transactions on Graphics (TOG), 32(6):206, 2013. DOI: $10.1145 / 2508363.2508399$.

[31] CA Weidner, Hoon Yu, Ronnie Kosloff, and Dana Z Anderson. Atom interferometry using a shaken optical lattice. Physical Review A, 95(4):043624, 2017. DOI: 10.1103/physreva.95.043624.

[32] PA Knott. A search algorithm for quantum state engineering and metrology. New Journal of Physics, 18(7):073033, 2016. DOI: 10.1088/1367-2630/18/7/073033.

[33] Robin Harper, Robert J Chapman, Christopher Ferrie, Christopher Granade, Richard Kueng, Daniel Naoumenko, Steven T Flammia, and Alberto Peruzzo. Explaining quantum correlations through evolution of causal models. Physical Review A, 95(4):042120, 2017. DOI: $10.1103 /$ physreva.95.042120.

[34] Rami Barends, Julian Kelly, Anthony Megrant, Andrzej Veitia, Daniel Sank, Evan Jeffrey, Ted C White, Josh Mutus, Austin G 
Fowler, Brooks Campbell, et al. Superconducting quantum circuits at the surface code threshold for fault tolerance. Nature, 508(7497):500-503, 2014. DOI: 10.1038/nature13171.

[35] CJ Ballance, TP Harty, NM Linke, MA Sepiol, and DM Lucas. High-fidelity quantum logic gates using trapped-ion hyperfine qubits. Physical review letters, 117 (6):060504, 2016. DOI: 10.1103/physrevlett.117.060504.

[36] Joseph M Renes, David Sutter, Frédéric Dupuis, and Renato Renner. Efficient quantum polar codes requiring no preshared entanglement. IEEE Transactions on Information Theory, 61(11):6395-6414, 2015. DOI: 10.1109/isit.2013.6620247.

[37] Lan Zhou and Yu-Bo Sheng. Purification of logic-qubit entanglement. Scientific reports, 6:28813, 2016. DOI: 10.1038/srep28813.

[38] Lan Zhou and Yu-Bo Sheng. Polarization entanglement purification for concatenated greenberger-horne-zeilinger state. Annals of Physics, 385:10-35, 2017. DOI: 10.1016/j.aop.2017.07.012.

[39] M Murao, MB Plenio, Sandu Popescu, V Vedral, and PL Knight. Multiparticle entanglement purification protocols. Physical Review $A, 57(6): \mathrm{R} 4075,1998$. DOI: $10.1103 /$ physreva.57.r4075.

[40] F Fröwis and W Dür. Stable macroscopic quantum superpositions. Physical review letters, 106(11):110402, 2011. DOI: 10.1103/physrevlett.106.110402.

[41] Florian Fröwis and Wolfgang Dür. Stability of encoded macroscopic quantum superpositions. Physical Review A, 85(5):052329, 2012. DOI: 10.1103 /physreva.85.052329.

\section{Supplementary Materials}

The software and additional online materials are available at qevo.krastanov.org and krastanov.github.io/qevo/index.html.

\section{A Model for operational errors}

We consider each two-qubit gate $\hat{U}$ to be performed correctly with a chance $p_{2}$ and to completely depolarize the two qubits $i$ and $j$ it is acting upon with chance $1-p_{2}$. Written as density matrices, when applied to input $\hat{\rho}_{i n}$ it results in

$$
\hat{\rho}_{\text {out }}=p_{2} \hat{U} \rho_{\text {in }} \hat{U}^{\dagger}+\left(1-p_{2}\right) \operatorname{Tr}_{i, j}\left(\hat{\rho}_{\text {in }}\right) \otimes \frac{\hat{I}_{i, j}}{4},
$$

where $T r_{i, j}$ is a partial trace over the affected qubits and $I_{i, j}$ is the identity operator associated with qubits $i$ and $j$. Similarly, measurement on qubit $i$ has a probability $\eta$ to properly project and measure and a probability $1-\eta$ to erroneously report the opposite result (flipping the qubit in the measurement basis). For instance, an imperfect projection on $|1\rangle$ reads as

$$
\hat{\rho}_{\text {out }}=\eta|1\rangle\left\langle 1\left|\rho_{\text {in }}\right| 1\right\rangle\langle 1|+(1-\eta)| 0\rangle\left\langle 0\left|\hat{\rho}_{\text {in }}\right| 0\right\rangle\langle 0| .
$$

Memory errors are not considered, but can be easily added to the optimization if required.

\section{B Purification example when opera- tions are interpreted as permutations of the Bell basis}

Consider the simple circuit from Fig. 1. As discussed throughout the main text, a useful way to represent the operations performed in the circuit is as permutations of the Bell basis. For this example we will use perfect operations (i.e. only initialization errors). The density matrix describing the system will be diagonal throughout the execution of the entire protocol as only permutation operations are performed. The following table describes how "mirrored" CNOT operation acts on the basis states ("AD" stands for "the sacrificial pair is in state $\mathrm{D}$ and the pair to be purified is in state A"): 


\begin{tabular}{|c|c|}
\hline initial state & mapped to \\
\hline \hline $\mathrm{AA}$ & $\mathrm{AA}$ \\
\hline $\mathrm{AB}$ & $\mathrm{DB}$ \\
\hline $\mathrm{AC}$ & $\mathrm{AC}$ \\
\hline $\mathrm{AD}$ & $\mathrm{DD}$ \\
\hline $\mathrm{BA}$ & $\mathrm{BC}$ \\
\hline $\mathrm{BB}$ & $\mathrm{CD}$ \\
\hline $\mathrm{BC}$ & $\mathrm{BA}$ \\
\hline $\mathrm{BD}$ & $\mathrm{CB}$ \\
\hline $\mathrm{CA}$ & $\mathrm{CC}$ \\
\hline $\mathrm{CB}$ & $\mathrm{BD}$ \\
\hline $\mathrm{CC}$ & $\mathrm{CA}$ \\
\hline $\mathrm{CD}$ & $\mathrm{BB}$ \\
\hline $\mathrm{DA}$ & $\mathrm{DA}$ \\
\hline $\mathrm{DB}$ & $\mathrm{AB}$ \\
\hline $\mathrm{DC}$ & $\mathrm{DC}$ \\
\hline $\mathrm{DD}$ & $\mathrm{AD}$ \\
\hline
\end{tabular}

With this mapping we can trace how the state of the system evolves. The following table gives the diagonal of the density matrix describing the system at each step. In the table $q=\frac{1-F}{3}$. The measurement column assumes a successful coincidence measurement has been performed. By normalizing and tallying the states that remain in A (for the purified pair) we are left with fidelity after purification $F_{\text {final }}=\frac{F^{2}+q^{2}}{F^{2}+5 q^{2}+2 F q}>F$.

\begin{tabular}{|c|c|c|c|}
\hline state & initial & after CNOT & final \\
\hline \hline $\mathrm{AA}$ & $F^{2}$ & $F^{2}$ & $F^{2}$ \\
\hline $\mathrm{AB}$ & $F q$ & $q^{2}$ & \\
\hline $\mathrm{AC}$ & $F q$ & $F q$ & \\
\hline $\mathrm{AD}$ & $F q$ & $q^{2}$ & $q^{2}$ \\
\hline $\mathrm{BA}$ & $F q$ & $q^{2}$ & $q^{2}$ \\
\hline $\mathrm{BB}$ & $q^{2}$ & $q^{2}$ & \\
\hline $\mathrm{BC}$ & $q^{2}$ & $F q$ & \\
\hline $\mathrm{BD}$ & $q^{2}$ & $q^{2}$ & $q^{2}$ \\
\hline $\mathrm{CA}$ & $F q$ & $q^{2}$ & $q^{2}$ \\
\hline $\mathrm{CB}$ & $q^{2}$ & $q^{2}$ & \\
\hline $\mathrm{CC}$ & $q^{2}$ & $F q$ & \\
\hline $\mathrm{CD}$ & $q^{2}$ & $q^{2}$ & $q^{2}$ \\
\hline $\mathrm{DA}$ & $F q$ & $F q$ & $F q$ \\
\hline $\mathrm{DB}$ & $q^{2}$ & $F q$ & \\
\hline $\mathrm{DC}$ & $q^{2}$ & $q^{2}$ & \\
\hline $\mathrm{DD}$ & $q^{2}$ & $F q$ & $F q$ \\
\hline
\end{tabular}

Table 1 gives more details on how different coincidence measurements act on the Bell pair.

\section{Purification example when opera- tions are interpreted as error detections}

\begin{tabular}{|c|c|c|c|c|}
\hline $\operatorname{coinX}$ & $\mathrm{Ao}$ & & $\operatorname{coin} Y$ & $\mathrm{Co}$ \\
\hline antiX & $\overline{\mathrm{B} o}$ & & antiY & $\mathrm{Ao}$ \\
\hline & & $\mathrm{nZ}$ & $A$ or $D$ & \\
\hline & & $\mathrm{iZ}$ & $\mathrm{B}$ or $\mathrm{C}$ & \\
\hline & & det & $\operatorname{cts} \mathrm{Y}$ al & \\
\hline & & & $\operatorname{cts} \mathrm{X}$ al & \\
\hline & & & $\operatorname{cts} \mathrm{X}$ ar & $\mathrm{Y}$ \\
\hline
\end{tabular}

Table 1: Coincidence and Anticoincidence Measurements. The three tables at the top show which two Bell states are selected by different Bell measurements. The measurements that select $A$, the state we are distilling, are highlighted. The other 3 possible coincidence measurements do not select for A so they are not highlighted, nor used as building blocks for our circuits. For the 3 measurements preserving $A$, the bottom table restates the same information in terms of what single qubit errors (I, X, Y, or Z Pauli operations) are detectable by each (if we have started in state $A$ ).

Another way to interpret the purification protocols is to look at them as error detection protocols. This way of thinking was used in the main text in the discussion of the limits imposed by the operational errors. Here we will repeat this discussion with more pedagogical visual aids for a particular choice of two-qubit operation and measurement. As in the main text, we will first consider a single selection circuit (where Alice and Bob share two Bell pairs and sacrifice one of them to detect errors on the other one). We are showing only Bob's side of the circuit.

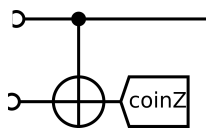

We assume that Alice and Bob started with two perfect Bell pairs in the state A. Each of the two registers (the one Alice uses to store her two qubits and the one Bob uses for his) are subject to complete depolarization with probability $\varepsilon=$ $1-p_{2}$. This is equivalent to saying that for each of the registers there is probability $\frac{\varepsilon}{16}$ for one of the 16 two qubit Pauli operators to be applied to the state. Writing the possibilities down in a table (columns correspond to the possible errors on the top/preserved qubit, rows correspond to the possible errors on the bottom/sacrificial, and each cell gives the corresponding tensor product): 


\begin{tabular}{|c|c|c|c|c|c|}
\hline & \multicolumn{4}{|c|}{ on preserved } \\
\hline & & I & $\mathrm{X}$ & $\mathrm{Y}$ & $\mathrm{Z}$ \\
\hline \multirow{4}{*}{ 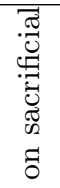 } & I & II & XI & YI & ZI \\
\hline & $\mathrm{X}$ & IX & XX & YX & ZX \\
\hline & $\mathrm{Y}$ & IY & $\mathrm{XY}$ & YY & $\mathrm{ZY}$ \\
\hline & $\mathrm{Z}$ & IZ & $\mathrm{XZ}$ & $\mathrm{YZ}$ & $\mathrm{ZZ}$ \\
\hline
\end{tabular}

If we are to perform a coincidence measurement immediately, we will be able to detect the errors that have occurred on the sacrificial qubit, however they are not correlated with errors that have occurred on the preserved qubit, therefore no errors on the preserved Bell pair would be detected. However, if we perform a CNOT gate, errors on either qubit will be propagated to the other one, and we will be able to detect some of the errors that have occurred on the Bell pair to be preserved by measuring the sacrificial Bell pair. Bellow we describe how the errors propagate:

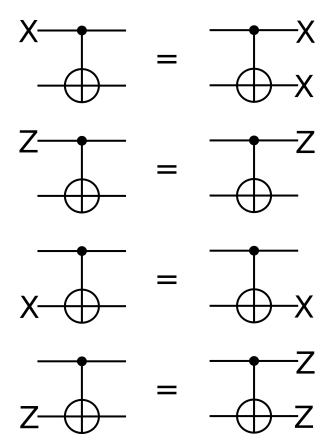

After the CNOT gate we have the following redistribution of errors:

\begin{tabular}{|c|c|c|c|c|c|}
\hline & \multicolumn{4}{|c|}{ on preserved } \\
\hline & & I & $\mathrm{X}$ & $\mathrm{Y}$ & $\mathrm{Z}$ \\
\hline \multirow{4}{*}{ 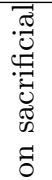 } & $\mathrm{I}$ & II & XX & YX & $\mathrm{ZI}$ \\
\hline & $\mathrm{X}$ & IX & XI & YI & ZX \\
\hline & $\mathrm{Y}$ & $\mathrm{ZY}$ & YZ & $\mathrm{XZ}$ & IY \\
\hline & $\mathrm{Z}$ & $\mathrm{ZZ}$ & YY & $\mathrm{XY}$ & IZ \\
\hline
\end{tabular}

Performing a coincidence $\mathrm{Z}$ measurement on the sacrificial Bell pair will be able to detect $X$ or Y errors, which leaves us with the following table conditioned on successful measurement.

\begin{tabular}{|c|c|c|c|c|c|}
\hline & \multicolumn{4}{|c|}{ on preserved } \\
\hline & & I & $\mathrm{X}$ & $\mathrm{Y}$ & $\mathrm{Z}$ \\
\hline \multirow{4}{*}{ 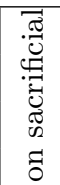 } & I & II & & & ZI \\
\hline & $\mathrm{X}$ & & XI & YI & \\
\hline & $\mathrm{Y}$ & & $\mathrm{YZ}$ & $\mathrm{XZ}$ & \\
\hline & $\mathrm{Z}$ & $\mathrm{ZZ}$ & & & IZ \\
\hline
\end{tabular}

Out of the 8 possibilities (16 initially), 2 (II \& IZ) are harmless to the preserved Bell pair and the remaining 6 are damaging, which leaves us with infidelity, to first order, $\frac{6}{16} \varepsilon \times 2$ (the factor of 2 comes from the fact that both Alice and Bob are subject to depolarization errors).

We can now augment the circuit with another level of detection that will be able to detect the $\mathrm{Z}$ error on the sacrificial Bell pair:

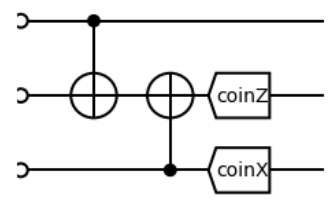

To first order any errors contributed by this extension are negligible and can not propagate back to the preserved Bell pair. The $\mathrm{Z}$ error that might have occurred on the middle line (and was left undetected) will now propagate to the bottom line and be detected by the coincidence $\mathrm{X}$ measurement leaving us with the following table:

\begin{tabular}{|c|c|c|c|c|c|}
\hline & \multicolumn{4}{|c|}{ on preserved } \\
\hline & & I & $\mathrm{X}$ & $\mathrm{Y}$ & $\mathrm{Z}$ \\
\hline.$]$ & I & II & & & ZI \\
\hline $\mathscr{G}$ & $\mathrm{X}$ & & $\mathrm{XI}$ & YI & \\
\hline  & $\mathrm{Y}$ & & & & \\
\hline శี & $\mathrm{Z}$ & & & & \\
\hline
\end{tabular}

Out of the 4 undetected errors, 3 still harm the preserved Bell pair, so we are left with fidelity $\frac{3}{16} \varepsilon \times 2$. Those three are undetectable as they do not propagate to the sacrificial qubits (they act as the identity on the sacrificial qubits). As such, using bigger registers (wider circuits) would provide only small higher-order corrections.

Finally, the asymptote reached by our circuits contains one additional source of infidelity. The black vertical lines in Fig. 5 correspond to short circuits with zero initialization error. However, a real purification protocol would need multiple rounds of purification until it lowers the non-zero initialization error to the steady-state floor governed by the operational error. In this steady state an additional round of purification would be able to detect only 2 of the possible 3 Pauli error that were already present, therefore raising the bound of the achievable infidelity from $\frac{3}{16} \varepsilon \times 2$ to $\frac{3+1}{16} \varepsilon \times 2$ (to first order in $\varepsilon$ ). For the parameters of Fig. 5 this would correspond to an 


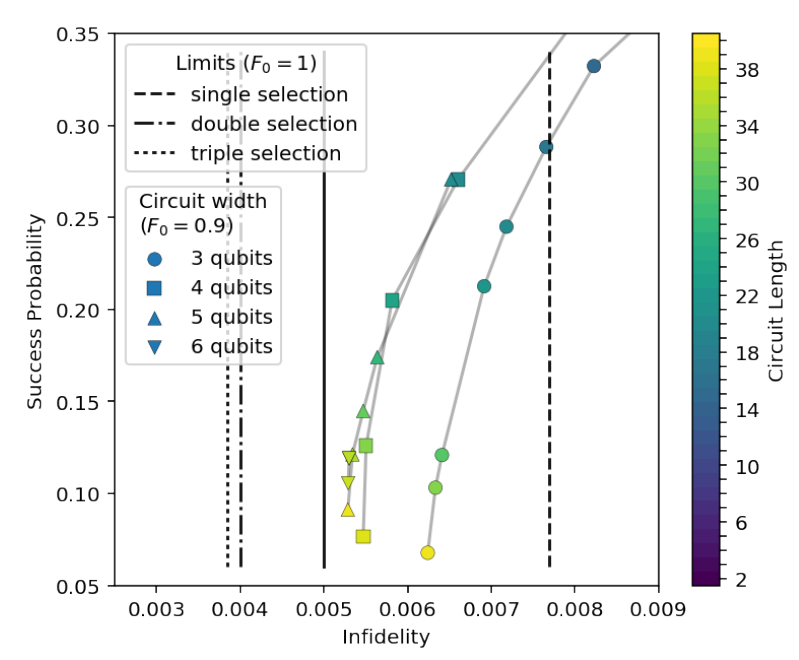

Figure 5: Same as 4 but we add some additional information. Dashed vertical lines, corresponding to perfectly initialized $\left(F_{0}=1, p_{2}=\eta=0.99\right)$ short purification circuits, are shown as a guide to how well the circuits perform in terms of initialization versus operational errors as described in the main text. In "single selection" circuits each party uses registers of size two, size three for "double selection", and size four for "triple selection".

asymptote at infidelity of 0.005 which is indeed what we observe.

The vertical lines of Fig. 5 are slightly offset from the values quoted above because we used exact numerics for the plots, as opposed to the first-order calculations of this section.

\section{Shortest multi-pair purification cir- cuits}

In the main text we introduced circuits to be used as benchmarks of initialization-vs-operation errors. The idea was to show what performance is provided by a circuit applied to perfectly initialized raw Bell pairs, or in other words, how much damage is caused by operation errors if we start with perfect initialization (as done in Fig. 4 and Fig. 7). To do that we found with bruteforce enumeration the best "short" circuits, i.e. circuits that do not reinitialize any of the consumed Bell pairs. They are named in the manner introduced in [22]. The triple select circuit is actually a generalization of the circuit from [22]. As described in [22] and in the main text of our manuscript, double selection significantly outperforms single selection, and extending the double selection circuit to a triple selection circuit provides only modest higher-order improvements.
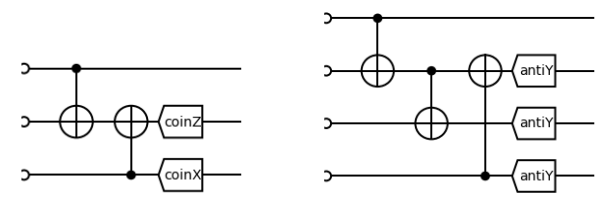

Figure 6: A double selection circuit on the left and a triple selection circuit on the right. We show only Bob's side of the circuit. The circuit from Fig. 1 can be referred to as a single selection circuit. As explained in the main text, there are many circuits with equivalent performance, related to the given circuits by permutation of the Bell basis.

\section{E More about initialization errors, op- erational errors, and the length of the circuit}

In Fig. 4 the vertical lines showed the "operational error" limit which one would reach if there were no initialization errors.

To make the comparison between initialization and operational errors clearer we provide Fig. 7 which drops the "success probability" axis of Fig. 4 and instead shows how the performance varies with $p_{2}$. In it one can see that the initialization infidelity is not a limiting factor - as long as the operational infidelity can be lowered, we can find longer circuits that iteratively get rid of the initialization error. For a sufficiently long circuit we reach a point of saturation, where, as described above, the operational error in the last operation dominates the infidelity of the final Bell pair. Similarly, if the circuit is wider (i.e. the register is bigger) we can obtain higher final fidelities for a fixed operational error, and the point saturation occurs at even lower operational error levels.

\section{F Circuits for registers with dedicated communication qubit}

Some hardware implementations of quantum registers have constraints on the two-qubit operations they can perform. For instance, only a single "communication" qubit might be able to establish a raw Bell pair with the remote register. Works like [7] suggest purification circuits specifically designed to fulfill such constraints. To see how our optimization protocol compares to 

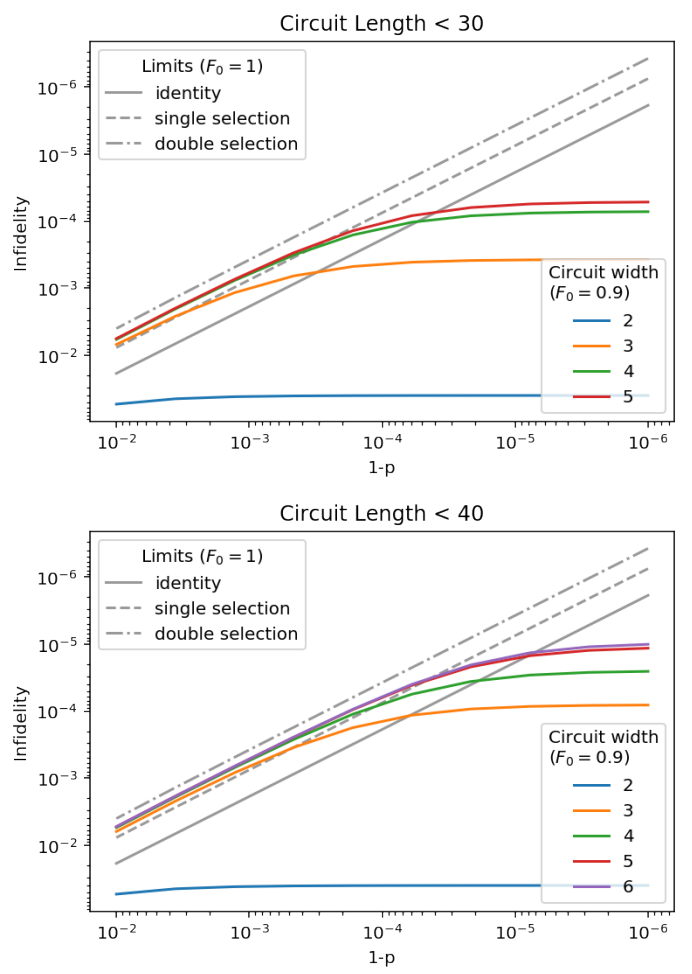

Figure 7: For each family of generated circuits of various width (color) and for a given operational infidelity ( $x$ axis) we show the best achievable final Bell pair fidelity by a circuit in that family. The top plot limits the permitted circuits to length less than 30 operations, and there is a limit of less than 40 operations for the bottom plot. Three important observations can be made: (1) as long as we can lower the operational error, we can design a long enough circuit that is not affected by the initialization error; (2) a wider register outperforms smaller registers and reaches a point of diminishing returns at smaller operational errors; (3) as already mentioned, circuits of width 2 are insufficient for arbitrary suppression of the initialization error as they detect only 2 of the possible 3 single-qubit errors ( $\mathrm{X}, \mathrm{Y}$, and $\mathrm{Z}$ ). The grey lines follow the same conventions as in Fig. 4 short perfectly initialized circuits used as a benchmark. The triple selection circuit from Fig. 4 is omitted as it can not be distinguished from the double select circuit on this scale. The "identity" line corresponds to what would happen if we simply depolarize a single perfect Bell pair with probability $1-p$.
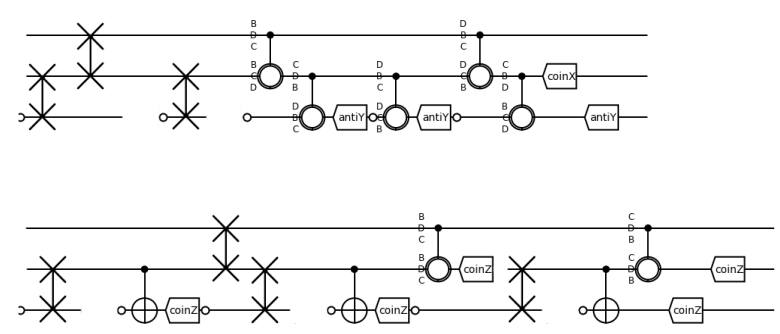

Figure 8: The top circuit is the one produced by our modified algorithm, while the bottom one is from Nigmatullin et al. [7]. We use the same notation as in the rest of the manuscript, in particular the small circles represent the reestablishment of a raw Bell pair (which here is only possible on the bottom-most qubit, i.e. the communication qubit). Our circuit requires only 5 raw Bell pairs (as opposed to 6 for the circuit from [7]), produces a final Bell pair of infidelity of $1.77 \%$ (as opposed to $2.46 \%$ ), at a success rate of $48 \%$ (as opposed to $43 \%$ ). The optimization and evaluation was done for $F_{0}=0.9$, $p_{2}=\eta=0.99$, without memory errors or errors from single-qubit unitaries. As discussed in the rest of the text, another circuit could be optimal for a different error model.

such hand-crafted circuits we made the following modifications: SWAP gates were added to the set of permitted operations; two-qubit gates were made available only on nearest neighbors; measurements on anything but the communication qubit became destructive; a reset operation (production of a new raw Bell pair) was added as a possibility, but only for the communication qubit. For the error regime we considered we were able to find a shorter circuit with higher fidelity and similar success probability as seen in Fig. 8.

\section{$G$ Different results when optimizing in different regimes}

In Fig. 9 we demonstrate the importance of optimizing your purification circuit for the exact hardware at which it would be ran. One can see that each of the three circuits outperforms the other two, only within a small interval around the parameter regime for which it was optimized.

\section{H Infidelity axes}

Even if the error model for the circuits and initialization is the depolarization model, the error in the final result of the purification needs not be 


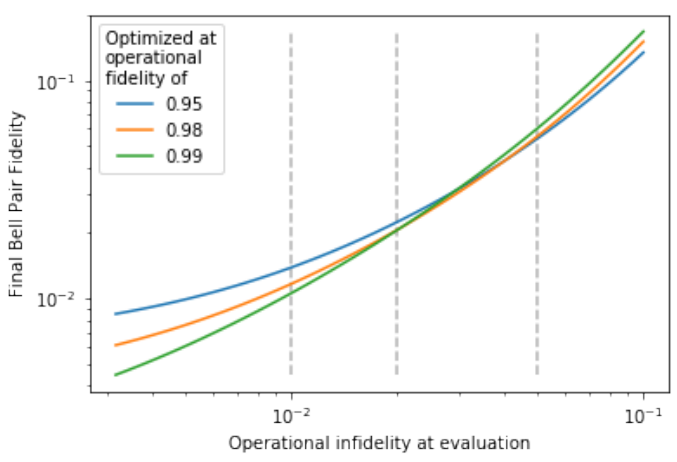

Figure 9: Each colored line corresponds to the result of a circuit optimization ran the given $p=p_{2}=\eta$ (depolarization noise). Then each of these circuits are evaluated at various values of $p$, different from the one they were optimized for. The $\mathrm{x}$ axis is the value of $1-p$ at evaluation. The $y$ axis is the obtained value of $1-F$. The dashed lines mark the values at which the optimizations were ran. The length of the circuits was constrained to $\leq 12$, the width was 3 and $F_{0}=0.9$.
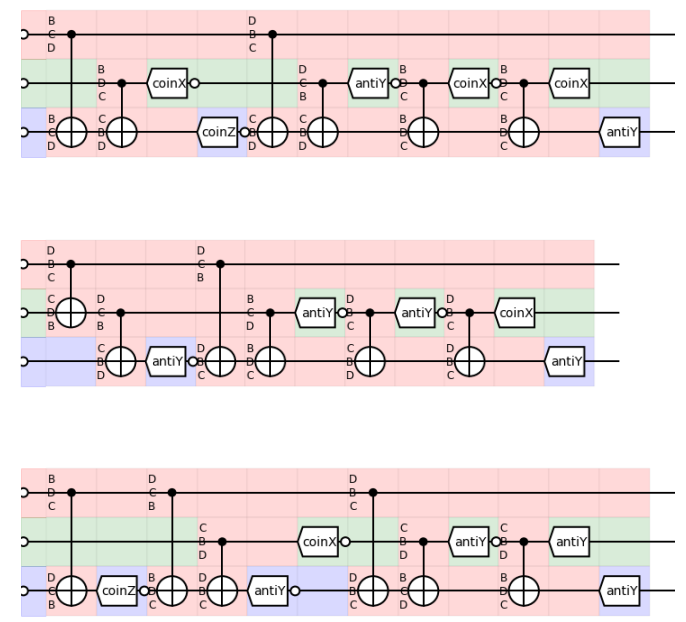

Figure 10: Top to bottom, the $0.95,0.98$, and 0.99 circuits from Fig. 9
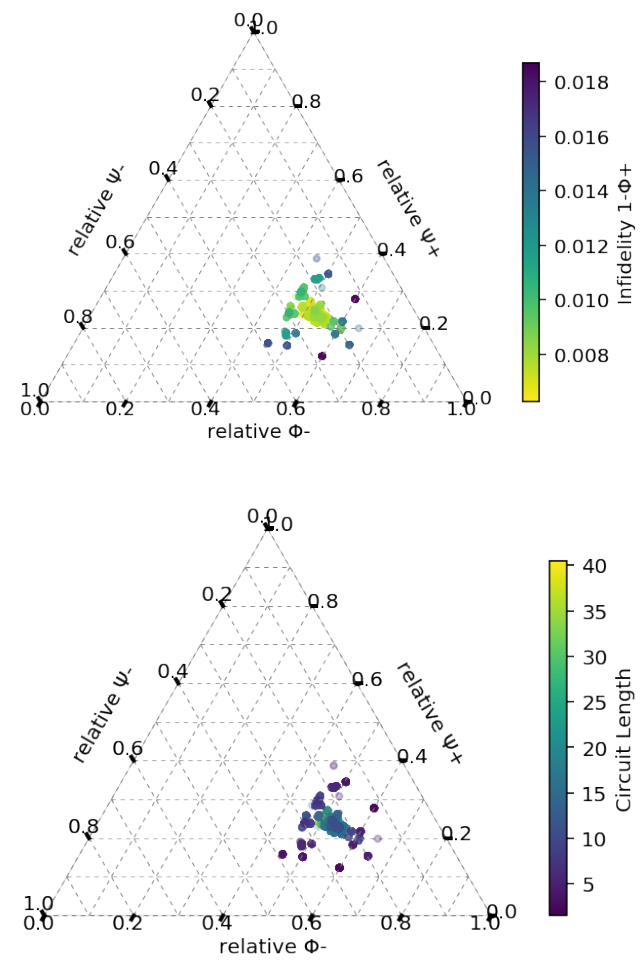

Figure 11: Each point corresponds to one of the circuits shown in Fig. 2. In the top plot they are colored by the final infidelity of the Bell pair produced by the circuit. In the bottom is the same plot, but the color corresponds to the length of the circuit. The 3 axes of the ternary plot correspond to the 3 components of the infidelity. As ternary plots require the 3 coordinates to fulfill a constraint, we plot the relative infidelity. For instance, the left axis (corresponding to the height of a point in the plot) shows the probability to be in $\psi_{+}$divided by the total probability to be in a state different from $\phi_{+}$ (the state being purified). Being in the center of the triangle means the infidelity in the final result is pure depolarization. Being in one of the corners means that one of the infidelity components dominates the other two. Being near the midpoint of a side means one of the infidelity components is much smaller than the other two. The 6 symmetries of this triangle correspond to the six permutations of $\{B, C, D\}$. 
depolarization. The infidelity in the final result has three components - the probabilities to be in states $\psi_{-}, \psi_{+}$, and $\phi_{-}$, respectively. Different purification circuits affect the three infidelity components differently, and giving different weights in the cost function of the optimization algorithm might be important, depending on the goal (for instance, if the purified Bell pairs are used for the creation of a GHZ state, the particular implementation might be more susceptible to phase errors, in which case that component would be assigned a higher weight). In Fig. 11 we show the distribution of the infidelity components of the purified Bell pair for each of the circuits we have generated. Of note is that longer circuits reach nearer the pure depolarization error, by virtue of lowering the infidelity to the level of diminishing returns where the depolarization from to the final operation dominates. Moreover, the results are biased to one particular type of error, due to the particular choice of "genes" described in the main text, namely, the $\{B, C, D\}$ permutations are performed before the CNOT gate and measurements. This bias can be removed if necessary by applying one final $\{B, C, D\}$ permutation (the six $\{B, C, D\}$ permutations correspond to the six symmetries of the triangle in Fig. 11).

\section{Implementation of the various per- mutation operations}

Here we give explicitly what single-qubit Clifford operations are necessary in order to perform a permutation of the Bell basis. $H$ stands for the Hadamard gate and $P$ states for the phase gate (in parenthesizes we mark whether the permutation is a rotation or a reflection of the triangle).

\begin{tabular}{|c|c|c|}
\hline permutation & Alice does & Bob does \\
\hline \hline BCD & nothing & nothing \\
\hline BDC(refl) & $H$ & $H$ \\
\hline DCB $($ refl $)$ & $H P H$ & $P H P$ \\
\hline CDB $($ rot $)$ & $P H$ & $(H P)^{2}$ \\
\hline DBC(rot) & $(P H)^{2}$ & $(H P)^{4}$ \\
\hline CBD $($ refl $)$ & $H(P H)^{2}$ & $H(H P)^{4}$ \\
\hline
\end{tabular}

Even though the decomposition of these operations in terms of $\mathrm{H}$ and $\mathrm{P}$ has different lengths, in practice these operations are equally easy to implement on real hardware.

\section{J Canonicalization of generated cir- cuits}

Many redundancies can appear in the population of circuits subjected to simulated evolution. To simplify the analysis of the results we filter the generated circuits by first ensuring that for each circuit:

- the first operation is not a measurement;

- does not contain two immediately consecutive measurements on the same qubit;

- does not contain unused qubits;

- does not contain measurement and reset of the top-most qubit pair (the one containing the Bell pair to be purified);

- does not contain non-measurement as a last step;

and then for the non-discarded circuits we perform the following canonicalization:

- reorder the qubits of the register so that the qubit closest to the top-most qubit is the one to be measured last, the second closest is measured second to last, etc;

- if a two-qubit gate and a measurement commute (i.e. they affect different qubits of the register), reorder them so that the gate is always before the measurement (in an implementation of that circuit, those two operations will be executed in parallel);

- if two two-qubit gates affect different qubits, put the one that affects top-most qubits before the one that affects lower qubits (in an implementation of that circuit, those two operations will be executed in parallel).

The canonicalization rules are arbitrary and any other consistent set can be used, at the discretion of the software writer. However, they ensure that two circuits that are physically equivalent are not presented multiple times in the final result, substantially lowering the circuits that need to be evaluated. The set described above is not exhaustive, as there are other, more complex, equivalences that we have not considered. 

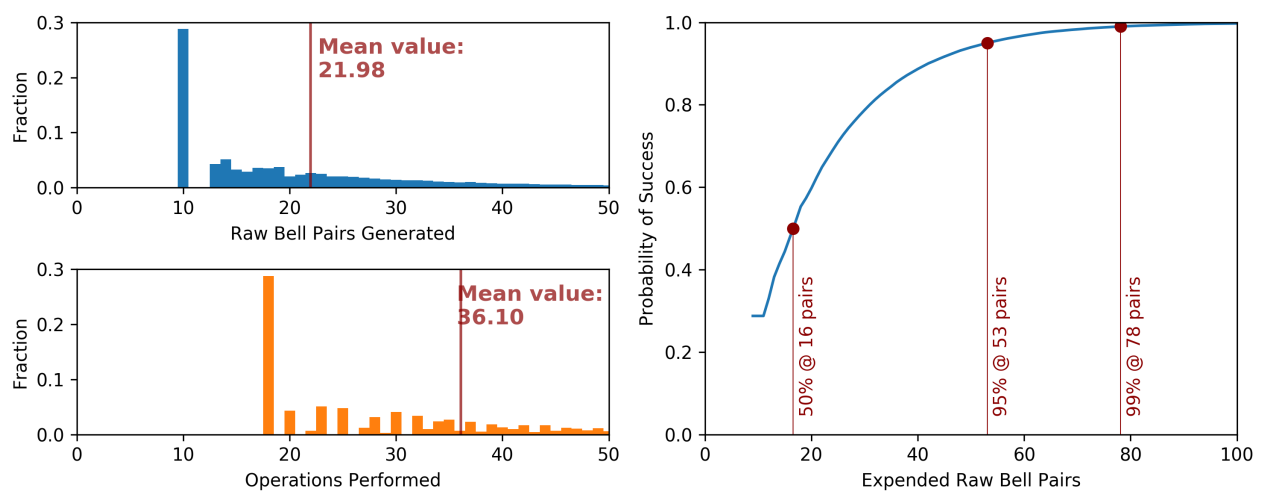

Figure 12: Monte Carlo evaluation of overhead due to restarts of failed measurements. The evaluation is from the circuit from Fig. 3 at $F_{0}=0.9$ and $p_{2}=\eta=0.99$. On the left we have the histogram of completed runs in terms of how many operations a run takes to successfully complete. In each histogram, the mean value of the distribution is showed as well. On the right we have the probability for the protocol (in which reinitializations are permitted) to successfully complete in terms of how many Bell pairs were used (i.e. it is the cumulative version of the top-left plot).

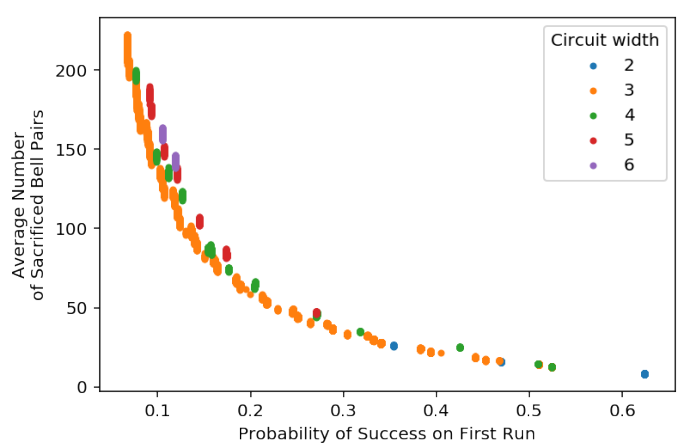

Figure 13: The relationship between overhead and success probability for the designs generated by our algorithm. Longer circuits have both lower success probability and higher overhead (expended Bell pairs). However, as shown in the rest of manuscript, longer circuits approach asymptotically the upper bound of performance.

\section{$\mathrm{K}$ Yield in the absence of measure- ment and operational errors}

As mentioned in the main text, if we concatenate a finite circuit with the hashing method, we can define a "hashing" yield even for our finite circuits. It is defined as $\frac{P}{N}(1-H(F))$, where $P$ is the success probability for the circuit under consideration, $N$ is the number of raw Bell pairs being sacrificed, $F$ is the fidelity of the Bell state produced by the circuit, and $H$ is the entropy in that Bell state (the information in that Bell state is the resource exploited by the hashing method to asymptotically reach unit fidelity, and consequently, $1-H\left(F_{0}\right)$, where $F_{0}$ is the initial



Figure 14: Plotted is the "hashing yield" of a given purification protocol versus the initial fidelity of the raw Bell pairs. The solid black line is the yield of the hashing method on its own. The dashed lines are the yields of the Oxford method (either on its own or used recursively) used as initial purification step and then continued by the hashing method. The colored lines are lower bounds for various protocols we have generated, used as initial purification step and then continued by the hashing method. The color represents the upper bound of how many raw Bell pairs the protocol requires. Of note is that although the hashing method on its own is the highest yield protocol when $F_{0} \approx 1$, around $F_{0} \approx 0.81$ we get $1-H\left(F_{0}\right)=0$ which requires the preprocessing (concatenation) steps, to bring the intermediate stage fidelity to a workable level. We do not optimize for the hashing yield, given that it is defined for perfect local gates and measurements. 
raw Bell pair fidelity, would be the yield one can achieve when using only the hashing method). This "hashing yield" definition faces a couple of problems in the parameter regime we work with. First, this quantity only refers to yield for circuits devoid of measurement and operational errors, but as we have seen, we need to consider such errors because the best finite purification circuit depends on the error model. Moreover, as our circuits might fail early, before all of the raw Bell pairs have been engaged, the factor $\frac{1}{N}$ is only a worst case bound. As such, the yield given by this expression is only a lower bound. We compare the yields given by the circuits we have generated to a number of known circuits in Fig. 14 (in the absence of measurement and operational errors). However, we remind the reader that the yield is not the cost function in our optimization algorithms, as our circuits are specifically optimized to deal with the aforementioned local errors.

\section{Analytical expressions for the final fi- delity}

Our software also produces a symbolic analytical expression for the fidelities obtained by each circuit. The quality of the raw Bell pairs is expressed as the quadruplet of probabilities to be in each of the Bell basis states $\left(F_{0}, q, q, q\right)$ where $q=\frac{1-F_{0}}{3}$ (more general non-depolarization error models are available as well). The purification circuit acts as a map that takes $(F, q, q, q)$ to the quadruplet $\left(F_{A}, b, c, d\right)$ representing the probabilities that the final purified Bell pair is in each of the Bell basis states.

The permutations of the Bell basis (i.e. all the local Clifford operations we are permitting) are polynomial maps, i.e. the output quadruplet contains polynomials of the variables in the input quadruplet. Depolarization is a polynomial map as well. Measurement without normalization is a polynomial map, but it becomes a rational function if normalization is required.

By postponing normalization until the very last step, we can use efficient symbolic polynomial libraries like Sympy (using generic symbolic expressions is much slower than using polynomials). The final result is given as a series expansion of the normalized expression (in terms of the small parameters $1-F_{0}, 1-p_{2}$, and $1-\eta$ ).

\section{Monte Carlo simulations of restart overhead}

As mentioned in the main text, one needs to consider how a protocol proceeds when a measurement fails. If there is a subcircuit that can be restarted, one needs not redo the entire proto$\mathrm{col}$, rather only reinitialize at the point where the subcircuit starts. However, if the top-most qubit pair, the one holding the Bell pair, is entangled with the qubit pair that undergoes a failed coincidence measurement, then the entire protocol has to be restarted. For most of our circuits, such subcircuits do not exist, but they are common among manually designed circuits. Our software automatically finds subcircuits and runs a Monte Carlo simulation of the sequence of measurements and reinitializations in order to evaluate the average resource usage as shown in Fig. 12.

The overhead estimated this way also proves to be closely related to the success probability of the given protocol, to be expected given that greater overhead implies more imperfect operations which implies higher chance of a fault (Fig. 13). 